\title{
Understanding the Molecular Machinery of Apoptosis
}

\section{Shaun Rosebeck*}

Department of Medicine, Biological Sciences Division, Hematology/Oncology Section, The University of Chicago, Chicago

\section{Introduction}

Apoptosis, or Programmed Cell Death (PCD), is a critical process whose proper regulation is essential to cellular and tissue homeostasis and is frequently perturbed during the course of autoimmunity, degenerative diseases, and neoplasia. Although conceptually first described in the 1800s, the cellular and histological changes associated with apoptosis were not differentiated from injurious cellular rupture until the 1960s and 1970s by seminal work from Kerr, Wyllie, and Currie. The term apoptosis was adopted from the Greek language wherein it refers to the falling off of petals from a flower or leaves from a tree, which represents the characteristic blebbing of cellular contents during apoptosis. Other morphological changes that occur during apoptosis include cell shrinkage, chromatin condensation, and exposure of the normally intracellular-facing lipid phosphatidylserine, the latter acting as a signal for phagocytosis by neighboring cells.

The first insights into the genetic mechanisms governing cell fate were described in the late 1980s by Horvitz and Ellis. These elegant studies identified key genes regulating organ development and PCD in a novel model organism, the nematode Caenorhabditis elegans, and, importantly, determined that these genes also exist in higher organisms, including man. As a testament to the impact of these studies, Horvitz was jointly awarded the 2002 Nobel Prize in Physiology or Medicine along with Brenner and Sulston, whose seminal work established C. elegans as an experimental system in which to interrogate the processes that regulate cell fate.

The work of Horvitz et al. identified two genes essential to the invariant death of specific cells during C. elegans development: CED-3 and CED-4. The death-inducing activity of these genes was found to be counteracted by another gene, CED-9, which was also found to be neutralized by EGL-1. This life/death axis thus laid the groundwork for an amazing flurry of discoveries that resulted in the identification and characterization of the cellular machinery responsible for dismantling a cell during apoptosis, which is defined both by these morphological changes and key molecular events that determine cellular commitment to apoptose.

Certain members of a family of cysteine-dependent aspartatedirected proteases, known as caspases, are responsible for cleaving intracellular substrates that promote the tidy destruction of a cell. Caspases, which are homologous to CED-3, are synthesized as inactive proteins whose activity is tightly regulated until certain conditions dictate that a cell must die. Stimuli that promote activation of these caspases originate either from within the cell through developmental cues or different types of stress, termed the intrinsic pathway, or from outside the cell via cognate death ligand-death receptor interactions, termed the extrinsic pathway.

When the intrinsic pathway is engaged, changes in mitochondrial morphology and integrity promote release of a key component of the electron transport chain known as cytochrome c. Upon its release into the cytoplasm, cytochrome $\mathrm{c}$ will bind to a protein complex composed of Apoptotic Protease Activating Factor 1 (Apaf-1), the human homolog of CED-4, and pro-caspase 9, the inactive form of the proteolytic enzyme. This complex then cleaves and generates the mature active form of caspase 9, which leads to activation of downstream effector caspases 3, 6, and 7 that then cleave the intracellular substrates whose proteolysis results in the morphological effects seen during apoptosis. Because of the key role of cytochrome $c$ and the subcellular site of insult, this process is also referred to as the mitochondrial pathway.

The extrinsic pathway is activated via cognate ligand engagement of cell-surface death receptors, many of which belong to the Tumor Necrosis Factor (TNF) receptor superfamily and include the Fas receptor, whose activity is essential for regulating proper lymphocyte homeostasis, and TNF Related Apoptosis Inducing Ligand (TRAIL), which triggers apoptosis in both lymphoid and non-lymphoid cell types. In contrast to the intrinsic pathway, death receptor engagement recruits pro-caspase 8 via associated factors, including Fas Associated Protein with Death Domain (FADD), leading to caspase 8 activation and effector caspase activation. In certain cell types, caspase 8 can also cleave the pro-apoptotic Bcl-2 family member Bid (tBid) which can subsequently promote activation of the mitochondrial pathway, serving to amplify the apoptotic signal.

To prevent unwanted execution of cell death, both pathways are tightly regulated. The B-cell lymphoma 2 (Bcl-2) family comprises three sub-families of apoptosis-regulating proteins. $\mathrm{Bcl}-2$ is the firstdiscovered member of the anti-apoptotic sub-family, which also includes Bcl-xL and Mcl-1, and is the human homolog of CED-9. These anti-apoptotic proteins serve as guardians against cytotoxic insult, but can be counteracted by the interplay of the other two pro-apoptotic Bcl-2 sub-families. Members of the Bax-like sub-family share structural similarity with $\mathrm{Bcl}-2$, particularly within the three conserved $\mathrm{Bcl}-2$ homology domains $(\mathrm{BH} 1, \mathrm{BH} 2$, and $\mathrm{BH} 3)$ and were identified by their ability to interact with $\mathrm{Bcl}-2$, which neutralizes their pro-apoptotic activity. The third sub-family contains many members, including Bid, Noxa, Puma, and Bim, that lack sequence similarity with Bcl-2 and each other, with the exception of the presence of a $\mathrm{BH} 3$ domain, and thus are referred to as the BH3-only sub-family, which are functionally analogous to the $\mathrm{C}$. elegans protein EGL-1. The BH3-only members function as damage sensors and antagonize the anti-apoptotic Bcl2 members, causing the release of the pro-apoptotic Bax-like subfamily members. This dynamic interplay occurs on the surface of the mitochondria and results in Bax sub-family-mediated permeabilization of the outer mitochondrial membrane, leading to release of cytochrome $\mathrm{c}$ and eventual activation of caspases (Figure 1).

One of the several stochastic characteristics of cancer cells is the

${ }^{*}$ Corresponding author: Shaun Rosebeck, PhD, Department of Medicine, Biological Sciences Division, Hematology/Oncology Section, The University of Chicago, KCBD 7116, 900 E 57 th St., Chicago, IL 60637; E-mail: srosebeck@medicine.bsd.uchicago.edu

Received December 14, 2012; Accepted December 19, 2012; Published December 20, 2012

Citation: Rosebeck S (2013) Understanding the Molecular Machinery of Apoptosis J Blood Lymph S3:e001. doi:10.4172/2165-7831.S3-e001

Copyright: (C) 2013 Rosebeck S. This is an open-access article distributed under the terms of the Creative Commons Attribution License, which permits unrestricted use, distribution, and reproduction in any medium, provided the original author and source are credited. 
ability to resist PCD. This is typically achieved either by perturbation of the sentinels that detect cellular abnormalities or stress, such as tumor suppressors, or by deregulated activation of proto-oncogenes. Perhaps one of the best-studied examples of tumor suppressors is TP53 or p53, which is typically activated in response to DNA damage. If the damage to DNA is irreparable, p53 will upregulate expression of $\mathrm{BH} 3$-only proteins and promotes apoptosis. TP53 is frequently lost or mutated in a wide variety of cancers, making these cells resistant to DNA damage-inducing therapeutics. Fortunately, clinical research has uncovered a number of approaches that circumvent the loss of p53, and several classes of drugs have been found to restore apoptosis sensitivity in TP53-mutant cancers (e.g. BH3 peptide mimetics and proteasome inhibitors).

One of the most commonly deregulated proto-oncogenes is c-myc. When mutated or deregulated, c-myc promotes expression of a large

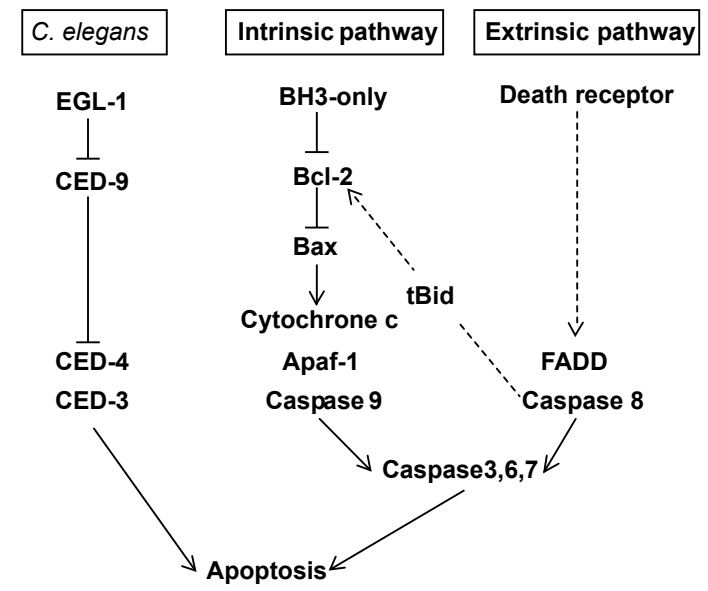

Figure 1: Overview of Apoptosis signaling (left).

In C. elegans, the first regulators of apoptosis were identified as necessary components governing cell fate in the developing organism. (middle and right). In higher organisms, apoptosis can be induced via the intrinsic and the extrinsic pathways. In the intrinsic pathway, pro-apoptotic $\mathrm{Bcl}-2$ sub-family members impair the protective effects of anti-apoptotic Bcl-2 sub family members and promote mitochondrial destabilization, release of cytochrome $c$ and effector caspase activation. The extrinsic pathway is activated via death ligand/death receptor-mediated activation of caspase 8 , which can either directly activate effector caspases or cleave Bid (tBid) to induce the intrinsic pathway. number of genes involved in proliferation, differentiation, and evasion of PCD. c-myc is a global regulator of gene transcription making it difficult, if not impossible, to specifically target and inhibit its activity in cancer cells while sparing normal cells. To overcome this type of obstacle, it is predicted that synthetic lethality strategies will be used to identify critical functional companions of c-myc that can be targeted for therapeutic intervention and may help to limit c-myc activity.

The last two decades have seen an explosion in our understanding of the mechanisms of apoptosis and how these pathways can be deregulated during the pathogenesis of cancer and autoimmune and degenerative diseases. Research should now be aimed at efficiently translating this growing body of knowledge into targeted therapies that can overcome the array of oncogenic and pathogenic perturbations responsible for the disease.
This article was originally published in a special issue, Molecular Mechanisms of Apoptosis handled by Editor(s). Dr. Shaun Rosebeck, The University of Chicago, USA 